\title{
Fractional Bloch Oscillations in photonic lattices
}

\author{
G. Corrielli, A. Crespi, G. Della Valle, S. Longhi, R. Osellame \\ Istituto di Fotonica e Nanotecnologie - Consiglio Nazionale delle Ricerche and Dipartimento di Fisica - Politecnico di Milano, \\ Piazza Leonardo da Vinci, 32, I-20133 Milano, Italy \\ giacomo.corrielli@mail.polimi.it
}

\begin{abstract}
We present the photonic analogy of the Fractional Bloch Oscillations [1]: the oscillatory motion of interacting particles moving in a periodic potential, under the presence of a static force. The analogy is implemented with the propagation of classical light in a specially engineered photonic waveguides lattice, fabricated in fused silica substrate via femtosecond laser micromachining.
\end{abstract}

\section{Introduction}

Bloch oscillations (BOs), i.e., the oscillatory motion of a quantum particle in a periodic potential driven by a constant force, constitute one of the most striking and oldest predictions of coherent quantum transport in periodic lattices. [2] In natural crystals, BOs have never been observed because of dephasing effects. Solely with the advent of semiconductor superlattices and ultracold atoms, BOs have been observed for matter waves [3].

When interactions between particles compete with their mobility, novel dynamical behaviour can arise where particles form bound states and co-tunnel through the lattice. While particle interaction has been generally associated to BO damping, for few strongly interacting particles it was predicted that bound states undergo fractional BO at a frequency twice (or multiple) that of single-particle [4]. The observation of fractional BO is challenging in condensed-matter systems and up to now has not been achieved even in model systems.

Light transport in specially engineered waveguides arrays already demonstrated in the past years to be a very powerful tool for the visualization of hard-to-observe coherent quantum phenomena [5-8]. Here we report on the first observation of fractional BOs using a photonic lattice as a model system of a two-particle extended BoseHubbard Hamiltonian driven by a constant force. The experimental realization of such structures is allowed by the unique 3D fabrication features offered by femtosecond laser micromachining.

\section{Discussion}

One of the main ideas behind this experiment is that the temporal evolution of two particles in a 1D lattice can be mapped into the light propagation in a $2 \mathrm{D}$ waveguides array, where the field amplitude in the (m,n)-th guide equals the probability of finding the two particles of the original problem in the $m$-th and $n$-th lattice site respectively [9]. Notice that the positions of the main diagonal, where $m=n$, correspond to have the particles occupying the same site. According to this approach, we fabricated the photonic structure sketched in fig. 1, made of 225 waveguides arranged in a $15 \times 15$ squared array, with lattice parameter $d=19 \mu \mathrm{m}$.
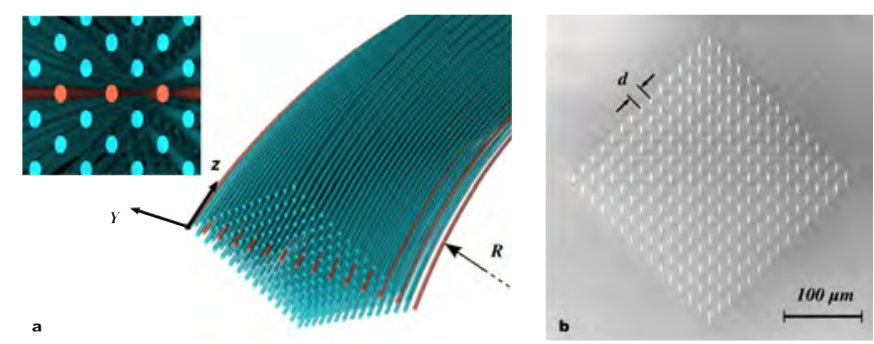

Fig. 1 a) Sketch of the photonic array fabricated for the visualization of the Fractional BOs. b) Section of the array. Picture taken with a optical microscope.

As already mentioned, the technique used for the fabrication is that of femtosecond laser micromachining. In particular, we used the second harmonic at $520 \mathrm{~nm}$ of a commercial $\mathrm{Yb}$ laser (comm. name: HighQ, from FemtoRegen, pulse length $=400 \mathrm{fs}$ ), used at $20 \mathrm{kHz}, 300 \mathrm{~nJ} /$ pulse, focused into a fused silica substrate with a 
microscope objective $(20 \mathrm{x}$, NA 0,45$)$. The direct writing of the waveguides with the desired geometry is obtained translating the substrate at a constant speed $=14 \mathrm{~mm} / \mathrm{s}$. The main diagonal (red-colored in fig. 1.a) is fabricated at a different velocity $=9 \mathrm{~mm} / \mathrm{s}$. This allows to change significantly the propagation constant $\left(\Delta \beta=4 \mathrm{~cm}^{-1}\right)$ with respect to that of the other waveguides, due to a different induced refractive index step. Since this latter quantity maps the depth of the potential wells of the lattice sites, it is possible in this way to take into account in our model system for the particle interaction. Moreover, the array is uniformly bent with a constant radius of curvature $\mathrm{R}=400 \mathrm{~cm}$, in order to add in our simulator the action of the constant force, responsible of the emerging of the oscillatory motion of the particles.

To carry out the experiment we focused with a $25 \mathrm{x}$ microscope objective a $633 \mathrm{~nm}$ He-Ne laser beam into the central waveguide of the array. Note that this waveguide belongs to the main diagonal and thus it corresponds to having the two particles perfectly localized in the same site at $\mathrm{t}=0$. We then observed the light distribution of the light into the array along a propagation length $\mathrm{L}=8,5 \mathrm{~cm}$. For this purpose we imaged from above (with a 10x microscope objective and a CCD camera) the fluorescence light that the waveguides written in fused silica with a femtosecond naturally emit, when excited with $633 \mathrm{~nm}$ radiation. The most relevant results obtained are shown in fig. 2 .

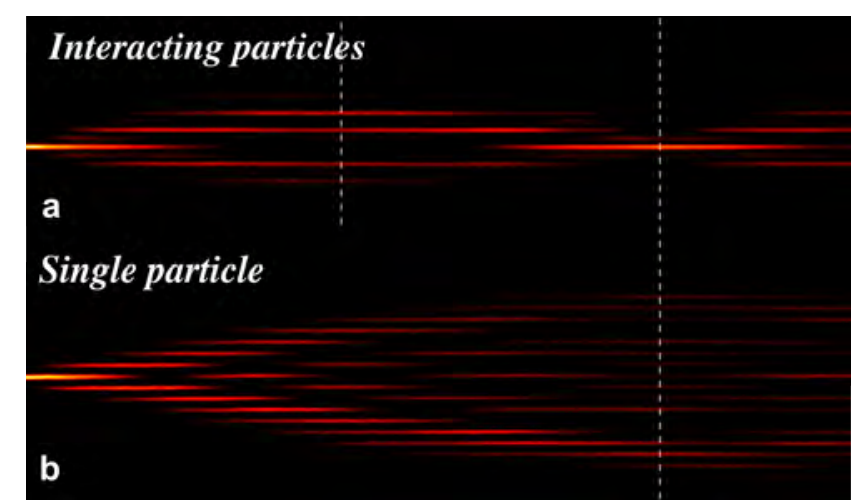

Fig.1 a) Photonic analogy of the fractional Bloch Oscillations for interacting particles. b) Photonic analogy of the Bloch oscillation of a single particle.

The periodic behavior visible in fig.2.a represents the so called "breathing mode", namely the Bloch Oscillations predicted theoretically for initially perfectly localized particles. The fractional nature of these oscillations is verified by fabricating a second array, that mimics the behavior of a single particle moving in the same array and under the action of the same force. It is clear from a direct comparison of fig. 2.a and 2.b that the oscillations of the interacting particles take place with a doubled frequency. Moreover, cutting and polishing the sample and collecting and imaging the light distribution at the array output facet at different lengths, we could prove that the light remains almost confined in the main diagonal along the propagation, and this confirms that the particles tend to form a bound state and to hop together in the lattice.

\section{Conclusions}

The presented experimental results validate the theoretical predictions of the extended Hubbard model about the motion of few strongly interacting particles moving in periodic potential, and represent the first observation of Fractional Bloch Oscillations. These results pave the way to the visualization of other intriguing phenomena involving few correlated particles, such as the interplay between particle interaction and Anderson localization among others.

\section{References}

[1] G. Corrielli et al, submitted (2012)

[2] F. Bloch, Z. Phys., 52, 555 (1928).

[3] M. Gustavsson et al, PRL, 100, 080404 (2008)

[4] W. S. Dias, Phys. Rev. B, 76, 155124 (2007).

[5] A. Szameit et al, Nature Physics, 5, 271-275 (2009)

[6] F. Dreisow et al, PRL, 102, 076802 (2009)

[7] A. Szameit et al, PRL, 104, 223903 (2010)

[8] A. Crespi et al, PRL, 108, 163601 (2012)

[9] S. Longhi, Optics Letter, 36, 015 (2011) 